\title{
$V$ and heavy flavour production: status and recent theoretical developments
}

\section{Davide Napoletano*}

IPhT, CEA Saclay, CNRS, Université Paris-Saclay, F-91191 Gif-sur-Yvette cedex, France.

E-mail: davide.napoletano@ipht.fr

\begin{abstract}
In this talk, we review the status of the associated production of a vector boson and heavy flavour quarks, and some recent theoretical developments. While some aspects of these multiscale processes are not yet completely under control, a general understanding on how to treat them is emerging, thanks to many efforts to match a completely massless, with a completely massive approach.
\end{abstract}

7th Annual Conference on Large Hadron Collider Physics - LHCP2019

20-25 May, 2019

Puebla, Mexico

${ }^{*}$ Speaker. 
The associated production of a vector boson $(V=W / Z / \gamma *)$ or a Higgs boson with heavy flavour quarks presents many interesting facets. From the experimental stand point, it constitutes irreducible backgrounds for many Standard Model measurements and beyond the Standard Model searches alike. From the theoretical stand point, it represents an important case study for multiscale problems. Indeed, as heavy quarks are mostly produced from a gluon splitting, a typical situation one may be in is

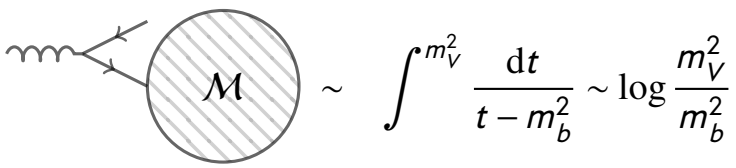

where $m_{V}$ in this case is just a place holder for, some, mass-like, hard scale. As it can be seen from Eq. (1), depending on the relative size of $m_{V}$ and $m_{b}$ one can get contributions that are enhanced by powers of the corresponding logarithm.

At this point, depending on the case at hand, one has the possibility of making two choices. One possibility consists in accepting the appearing logarithms (assuming they are small) as a fact of life to retain heavy quark mass effects. In order for this approach to work, one needs to define a scheme in which, with respect to QCD evolution, the mass of the heavy quark is effectively infinite, and thus decoupled. Such a scheme is commonly referred to as the Four Flavour Scheme (4FS) in the case of $b$-quarks, and clearly is a valid approximation when the scale of interest does not exceed by much the mass of the heavy quark. Alternatively, one can decide to neglect all mass effects, transforming the logarithm in Eq. (1) in a collinear divergence, and resum to all orders the contributions coming from it, in the same way as for all other light quarks, effectively defining a $b$-quark PDF. Such a scheme is called a Five Flavour scheme (5FS) and it is expected to be valid in the region where $m_{V} \gg m_{b}$.

Comparisons between these two approaches for the production of $b$-quarks in association with a vector boson, have shown that, overall, the most accurate $5 \mathrm{~F}$ prediction seems to favour data better. This has been supported by various inclusive calculations aiming at matching these two schemes: at scales relevant at the LHC, logarithmic effects are generally more important than mass power corrections[1, 2, 3, 4]. Nevertheless, there are differential observables for which including mass effects may still have an important impact. For these reasons various recent attempts to consistently include both the resummation of high energy logarithms and mass effects have been made.

Although not discussed in this talk, among the various efforts it is worth noting Ref. [5]. More recently a similar approach in spirit have been developed in the context of the SHERPA Monte Carlo generator, based on multi-jet merging [6]. A diagrammatic representation of how the multijet merging algorithm works in this case is presented in Fig. 1.

The working idea in this case is quite straightforward. Taking as an example the production of a $Z$ boson plus a pair of $b$-quarks, one starts by generating both an inclusive 5FS sample which corresponds to $Z+$ jets with multi-jet merging, and a calculation in the 4FS, $Z+b \bar{b}$. The next step consists in processing the latter in the same way as if it came from the former, by applying the normal clustering procedure. This generates what the authors call the direct component. The last step (fusing) consists in removing from the 5FS sample all events that have a configuration which 

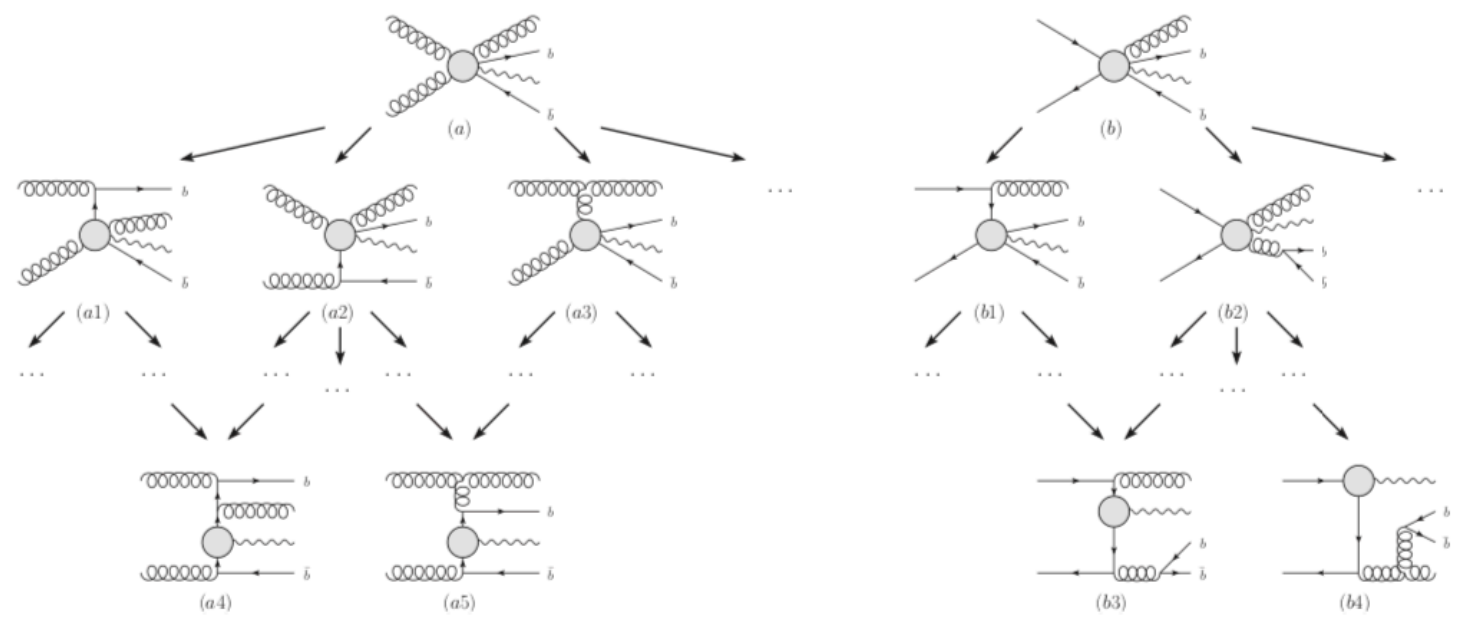

Figure 1: Pictorial description of the clustering for one the various processes contributing to the production of a $Z$ boson in association with heavy flavours.

can also be generated by the reclustered 4FS one. This creates what the authors refer to as the fragmentation component, and one obtains the fused result by combining the two samples.

The authors then go on and make some connection to the inclusive FONLL method. Here it has to be noted that while indeed they are able to construct some relevant terms necessary to construct the FONLL matching, the accuracy they get is only NLO plus that of the parton shower (which would be LL in this case). Conversely, even the lowest order FONLL matching, FONLL-A is NLO+NNLL[1, 4]. Nevertheless, the multijet-merging method is systematically improvable, by either including higher fixed order corrections, or corrections to the parton shower. Some relevant distributions are shown in Fig. 2, for the exampled described above.

An somewhat older and alternative approach, was first introduced in [7], and consists in extending the 5FS scheme to include mass effects even for initial state particles. This has the advantage to include the resummation of high energy logs, which are included in the $b$-PDF, as well as mass power corrections, which then enter in the matrix elements and phase-space. A the time of [7], however, this method suffered by two important limitations. First, it is known that calculations with initial state massive quarks suffer from higher-order uncancelled soft-divergence, which may render the NLO result useless. Second, it was not clear whether or not one was allowed to consistently use standard PDFs with massive initial states. As for the former issue, research to study to what extent this is an actual issue are ongoing.

The latter issue has instead recently been resolved [8]. Indeed it has been shown that a $5 \mathrm{~F}$ 

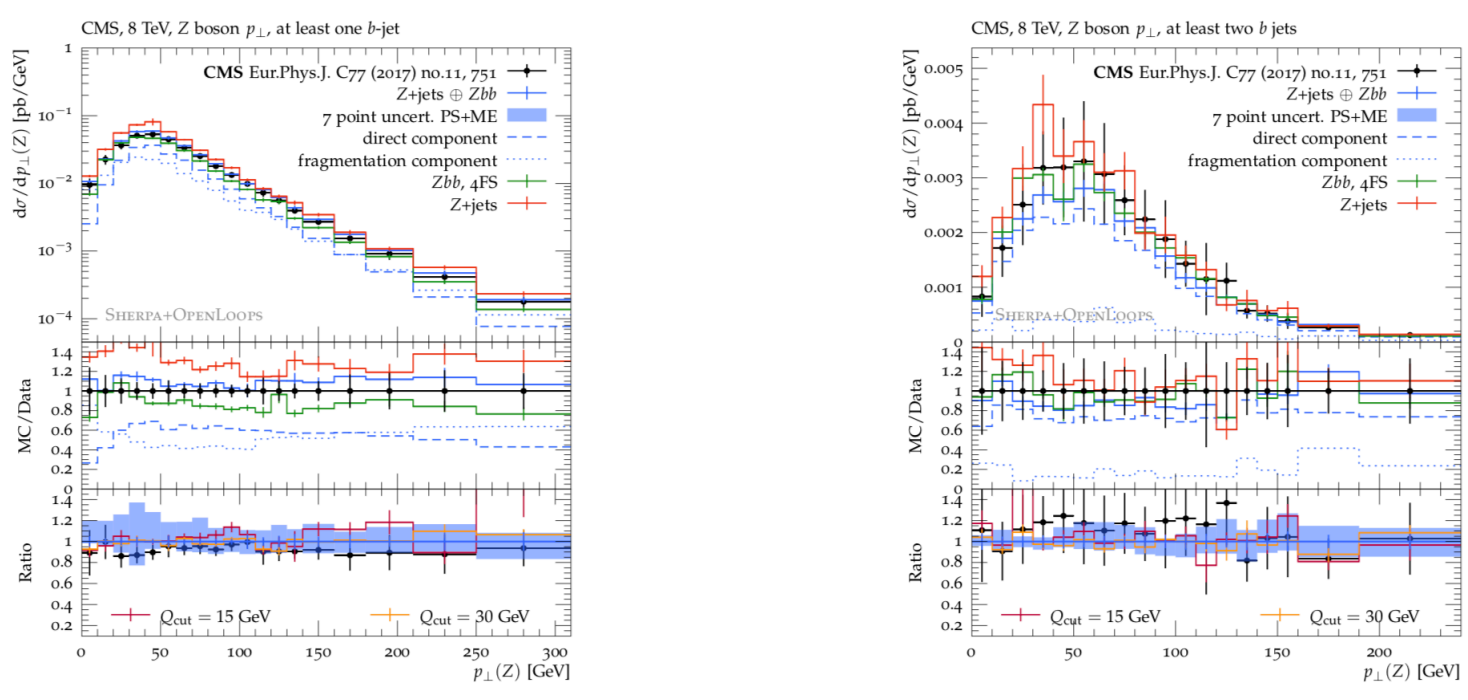

Figure 2: Comparison of the multijet merging (fused) scheme with the standard 5FS as well as with the components of the merged results. The left panel refers to an event selection where one has at least one $b$ jet, while in the right panel, a selection with at least $2 b$-jets is made.

massive scheme $e^{1}$ can be consistently construct by simply constructing a parametrised $b$-PDF, and performing the FONLL matching between the 4FS and the 5FS: the matched FONLL result is indeed identical to what is defined in Ref. [7]. The caveat is that one has to assume that, in some sense, some intrinsic component of the heavy flavour exists. However, this can be simply seen by lowering the heavy quark threshold. This already happens in standard PDF sets, where the $b$-PDF is given by matching conditions: starting at NNLO, constant terms in the matching conditions make the heavy quark PDF non-zero even below threshold.

Just as an example, we report in Fig. 3 results obtained for $b \bar{b} \rightarrow H$ in this scheme. On the left panel we show the independence of the matched cross section with respect to the heavy quark matching scale, while on the right hand side a comparison between the 5FS and the 5F massive (or FONLL) is shown as a function of the factorisation scale.

In conclusion, in this talk we have presented some recent theoretical developments needed to achieve a more accurate description of processes involving heavy flavours. This is, in particular, related to how one decides to treat initial state heavy quarks. Improvements at the inclusive and at the differential level are on-going. While the multi-jet merging approach presents various interesting aspects, it still needs some detail studies and comparisons, at least in the view of the authors. On

\footnotetext{
${ }^{1}$ Note that this is true for any heavy flavour scheme.
} 

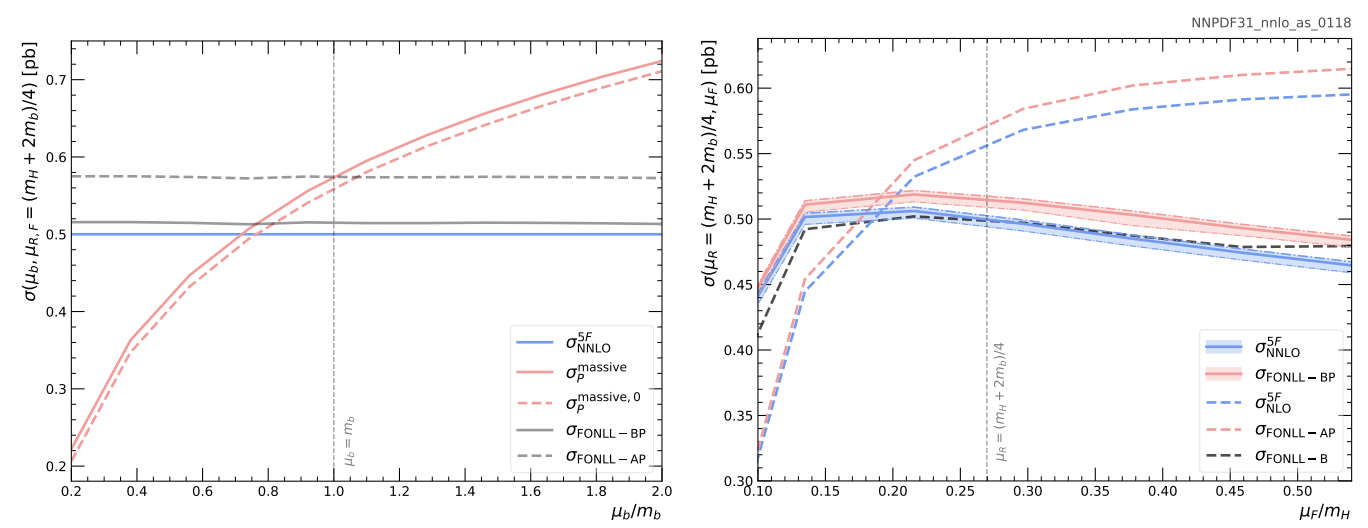

Figure 3: Results in the FONLL scheme, and comparison to the standard 5FS.

the other hand recent theoretical progresses in the matching of inclusive calculations, have shown to what extent the use of a massive heavy flavour scheme, or in other words a scheme in which heavy flavours are taken as massive even when in the initial state, is reliable. We hope that in the future fitted $b$-PDFs will be provided together with standard PDFs, as this will make the use of such schemes completely consistent.

\section{References}

[1] S. Forte, D. Napoletano and M. Ubiali, Phys. Lett. B 751 (2015) 331 doi:10.1016/j.physletb.2015.10.051 [arXiv:1508.01529 [hep-ph]].

[2] S. Forte, D. Napoletano and M. Ubiali, Phys. Lett. B 763 (2016) 190 doi:10.1016/j.physletb.2016.10.040 [arXiv:1607.00389 [hep-ph]].

[3] F. Krauss, D. Napoletano and S. Schumann, Phys. Rev. D 95 (2017) no.3, 036012 doi:10.1103/PhysRevD.95.036012 [arXiv:1612.04640 [hep-ph]].

[4] S. Forte, D. Napoletano and M. Ubiali, Eur. Phys. J. C 78 (2018) no.11, 932 doi:10.1140/epjc/s10052-018-6414-8 [arXiv:1803.10248 [hep-ph]].

[5] E. Bagnaschi, F. Maltoni, A. Vicini and M. Zaro, JHEP 1807 (2018) 101 doi:10.1007/JHEP07(2018)101 [arXiv:1803.04336 [hep-ph]].

[6] S. Höche, J. Krause and F. Siegert, Phys. Rev. D 100 (2019) no.1, 014011 doi:10.1103/PhysRevD.100.014011 [arXiv:1904.09382 [hep-ph]].

[7] F. Krauss and D. Napoletano, Phys. Rev. D 98 (2018) no.9, 096002 doi:10.1103/PhysRevD.98.096002 [arXiv:1712.06832 [hep-ph]].

[8] S. Forte, T. Giani and D. Napoletano, Eur. Phys. J. C 79 (2019) no.7, 609 doi:10.1140/epjc/s10052-019-7119-3 [arXiv:1905.02207 [hep-ph]]. 